Z. Klin. Chem. Klín. Biochem.

13. Jg. 1975, S. 75-77

\title{
Statistische Qualitätssicherung gerinnungsanalytischer Meßgrößen
}

\author{
Von H. Zöller, W. Gross und E. Moll \\ Aus der Medizinischen Poliklinik (Direktor: Prof. Dr. H. Franke) der Universität Würzburg
}

(Eingegangen am 19. September/7. Dezember 1974)

\begin{abstract}
Die von klinisch-chemischen Analysen her bekannte Qualitätssicherung durch Präzisions- und Richtigkeitskontrolle wird in ihrer Anwendbarkeit auf Gerinnungsuntersuchungen überprüft. Zur Beobachtung gelangen die Globaltests Partielle Thromboplastinzeit und Plasmathrombingerinnungszeit sowie die plasmatischen Gerinnungsfaktoren Fibrinogen (Faktor I) und Faktor VII/X. Dabei zeigt sich, daß eine Präzisionskontrolle bei gerinnungsanalytischen Meßergebnissen grundsätzlich möglich ist, wenngleich der Variationskoeffizient der Einzelfaktoren höher liegt als derjenige der Gruppentests. Eine Richtigkeitskontrolle, beruhend auf dem Vergleich von Istwert und Sollwert, ist nur bedingt durchführbar. Die Ursache hierfür liegt u. a. in der Schwierigkeit, absolute und konstante Referenzplasmen zu gewinnen und eine stabile Aktivität der verwendeten Reagenzien zu gewährleisten.
\end{abstract}

\section{Quality control in blood-clotting analyses}

Quality control of laboratory precision and accuracy was studied in coagulation analyses. The following were investigated: Partial-thromboplastin-time, plasma-thrombin-time, fibrinogen and clotting factor VII/X. It is evident that precision may be controlled both in global coagulation tests and factor analyses. Control of accuracy, i. e. the difference between true and obtained values must be regarded with reserve. This is due to the difficulties of producing absolute and constant standards and stabilizing the activity of clotting reagents.

Die chemische Analyse gleicher Kontrollproben durch verschiedene Laboratorien in sog. Ringversuchen ergab bis zu 40 Prozent fehlerhafte Ergebnisse (1, 2, 3). Die Zuverlässigkeit von Laborergebnissen muß deshalb durch eine laborinterne Präzisionskontrolle zur Erfassung zufälliger Fehler und eine Richtigkeitskontrolle zur Erfassung systematischer Fehler verbessert werden (s. Richtlinien der Bundesärztekammer (4)). Gerade hämostaseologische Untersuchungen sind besonders empfindlich gegenüber vielfältigen Störfaktoren wie Temperaturschwankungen, $\mathrm{pH}$-Verschiebungen, Verunreinigung der verwendeten Glaswaren, Konzentrationsverlust der Reagenzien etc. Im folgenden sollen die Möglichkeiten und Grenzen einer statistischen Qualitätskontrolle für globale Gerinnungstests und plasmatische Gerinnungsfaktoren an Hand einer Laborstudie untersucht werden.

\section{Methodik der Gerinnungsuntersuchungen}

Zur Untersuchung gelangen die Partielle Thromboplastinzeit, die Plasmathrombinzeit sowie die plasmatischen Gerinnungsfaktoren Fibrinogen (= Faktor I) und Faktor VII/X. Die Kontrollplasmen (lyophilisiertes Standard-Human-Plasma der Behringwerke) werden sofort nach der Lösung verwendet. Die Untersuchungen finden in Glasmaterial statt.

Für die Partielle Thromboplastinzeit werden $0,2 \mathrm{ml}$ Citratplasma mit $0,2 \mathrm{ml}$ kaolinaktiviertem Reagenz $2 \mathrm{~min}$ bei $37^{\circ} \mathrm{C}$ inkubiert und nach Zupipettieren von $0,2 \mathrm{ml} 0,02 \mathrm{~mol} / 1$ $\mathrm{CaCl}_{2}$-Lösung der Gerinnungseintritt mit der Häkchenmethode ermittelt (Methodik s. Perlick \& Bergmann (5); Reagenzien Behringwerke).
Für die Plasmathrombingerinnungszeit werden $0,1 \mathrm{ml}$ Citratplasma mit $0,1 \mathrm{ml}$ dest. Wasser $20-30 \mathrm{~s}$ bei $37^{\circ} \mathrm{C}$ inkubiert und mit $0,1 \mathrm{ml}$ verd. Thrombinlösung $(0,6 \mathrm{NIH}$-Units) versetzt. Der Gerinnungseintritt wird manuell mit der Häkchenmethode bestimmt (5) (Reagenzien Behringwerke).

Fibrinogen wird nach Clauss (6) durch Inkubation von $0,2 \mathrm{ml}$ 1:10 verd. Citratplasma über eine Minute bei $37^{\circ} \mathrm{C}$ und Zugabe von $0,2 \mathrm{ml}$ Thrombinlösung mittels Häkchenmethode gemessen. Die Aktivität wird an Hand einer Standard-Eichkurve abgelesen (Reagenzien Boehringer, Mannheim).

Faktor VII/X (nach Schultze \& Schwick, (7)) wird nach dem Prinzip des Einphasentests nach Quick mit artifiziellem Mangelplasma ermittelt: $0,1 \mathrm{ml} 1: 20$ verd. Citratplasma und $0,1 \mathrm{ml}$ Faktor X-Mangelplasma werden $30 \mathrm{~s}$ bei $37^{\circ} \mathrm{C}$ inkubiert und $0,2 \mathrm{ml} \mathrm{Ca-Thromboplastinlösung} \mathrm{zugegeben.} \mathrm{Der} \mathrm{mit} \mathrm{der} \mathrm{Häk-}$ chenmethode bestimmte Gerinnungswert wird an Hand einer Eichkurve in \%-Aktivität umgerechnet (Reagenzien Behringwerke).

\section{Ergebnisse}

\section{Präzisionskontrolle}

In einer Vorperiode werden an 20 aufeinanderfolgenden Tagen die Partielle Thromboplastinzeit, die Plasmathrombinzeit, Fibrinogen und Faktor VII/X an einer Präzisionskontrollprobe derselben Herstellungscharge (Standard-Human-Plasma, Behringwerke) gemessen und die folgenden Parameter errechnet $(8,9)$ : Mittelwert, Standardabweichung von Tag zu Tag, Warngrenzen bzw. Kontrollgrenzen $\bar{x} \pm 2 s$ bzw. $\bar{x} \pm 3 s$, Variationskoeffizient $\mathrm{V}(\%)$. 
Der Variationskoeffizient betrug für die Untersuchung von Tag zu Tag bei den Globaltests Plasmathrombinzeit 4,64\% und Partielle Thromboplastinzeit 5,48\%. Für die plasmatischen Gerinnungsfaktoren lag der Variationskoeffizient deutlich höher und zwar für Faktor VII/X bei 7,26\% und für Fibrinogen bei 8,18\% (Tab. 1).

In der eigentlichen Kontrollperiode wird eine weitere Kontrollkarte mit den in der Vorperiode ermittelten Grenzen $\bar{x} \pm 2 s$ und $\bar{x} \pm 3 s$ angelegt. Es wird an jedem Tag, an dem Analysen vorgenommen werden, ein frisch gelöster Standard derselben Charge für die Gerinnungsmeßwerte mitgefuihrt und in die Kontrollkarte eingetragen (Abb. 1). Je Analysenserie werden etwa 15-20 Patientenplasmen untersucht. Einmal während des Beobachtungszeitraumes von einem Vierteljahr geriet das System bei der Partiellen Thromboplastinzeit- und Fibrinogenmessung , außer Kontrolle“. Durch entsprechende Suche konnten die Fehlerursachen eliminiert werden. Verantwortlich waren Pipettierfehler bzw. eine Verunreinigung der Glaswaren.

Die Befunde zeigen, daß eine Präzisionskontrolle bei gerinnungsanaly tischen Untersuchungen möglich ist. Die Präzision von Tag zu Tag, ausgedrückt als Variationskoeffizient, darf nach Angaben der Bundesärztekammer bei Gerinnungsanalysen $10 \%$ nicht überschreiten (4).
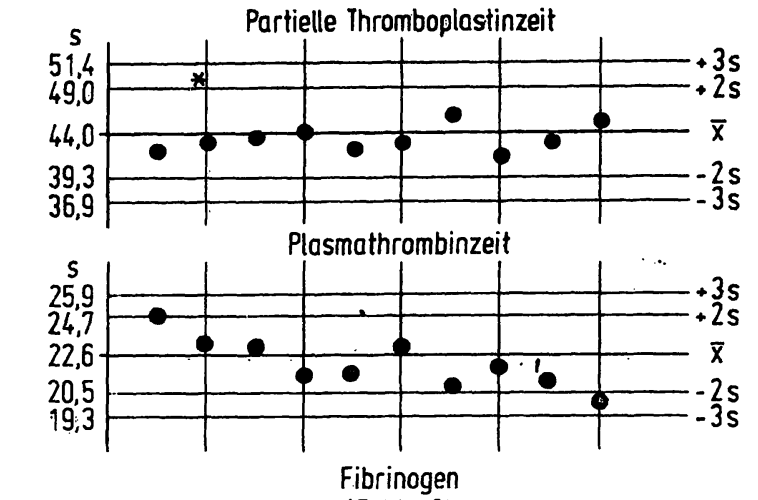

(FaktorI)
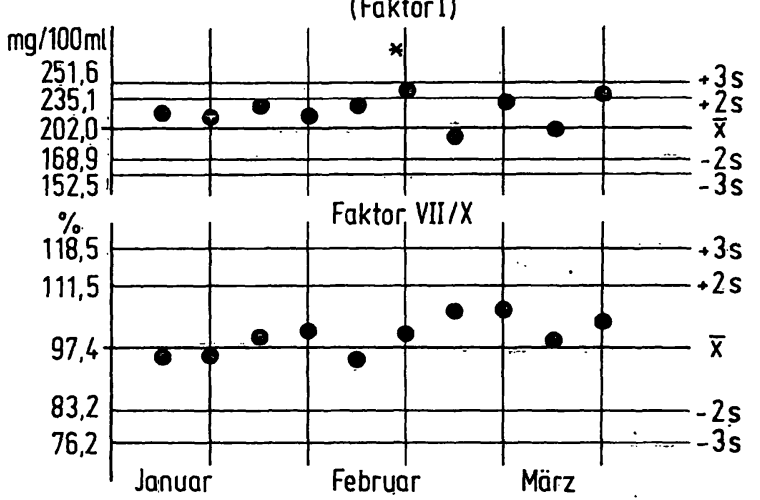

Abb. 1. Kontrollkarte für die Präżisionnskọntrolle von Partieller Thromboplastinzeit, Plassmathrombinzeit, Fibrinogen und Faktor VII/X während der Kontrollperiode. $*=$,Methode außer Kontrolle“", Meßwert nach TestWiederholung.

Tab. 1. Mittelwert, Standardabweichung, Variationskoeffizient und Kontrollgrenzen bei der Präzisionskontrolle der Gerinnungsparameter

\begin{tabular}{|c|c|c|c|c|c|c|}
\hline Meßgröße & Anzahl (n) & $\overline{\mathbf{x}}$ & $\mathbf{s}$ & V(\%) & $\overline{\mathrm{x}} \pm 2 \mathrm{~s}$ & $\bar{x} \pm 3 s$ \\
\hline $\begin{array}{l}\text { Plasmathrombinzeit (s) } \\
\text { Partielle Thromboplastinzeit (s) } \\
\text { Fibrinogen (mg/l) } \\
\text { Faktor VII/X (\%) }\end{array}$ & $\begin{array}{l}20 \\
20 \\
20 \\
20\end{array}$ & $\begin{array}{r}22,6 \\
44,1 \\
2020 \\
97,4\end{array}$ & $\begin{array}{r}1,1 \\
2,4 \\
165 \\
7,1\end{array}$ & $\begin{array}{l}4,6 \\
5,5 \\
8,2 \\
7,3\end{array}$ & $\begin{array}{r}20,5-24,7 \\
39,3-49,0 \\
1689-2351 \\
83,2-111,5\end{array}$ & $\begin{array}{r}19,3-25,9 \\
36,9-51,4 \\
1524-2516 \\
76,2-118,5\end{array}$ \\
\hline
\end{tabular}

Tab. 2. Kontrollbogen zur Richtigkeitskontrolle (Behringwerke) Plasmathrombingerinnungszeit

\begin{tabular}{|c|c|c|c|c|c|c|c|c|c|}
\hline 1 & 2 & 3 & 4 & & 5 & 6 & 7 & & \\
\hline Sollwert & $\begin{array}{l}\text { Analysen- } \\
\text { ergebnis }\end{array}$ & $(1)-(2)$ & + & - & $\begin{array}{l}\% \\
\text { Abweichung } \\
\text { vom Sollwert } \\
\frac{(3)}{(1)} \cdot 100= \\
(\max .20 \%)\end{array}$ & $3 \times \mathrm{V}(\%)$ & $\begin{array}{l}\text { Üb } \\
\text { aus }\end{array}$ & $\begin{array}{l}\text { timmung } \\
\text { nd } \\
\text { nein }\end{array}$ & Datum \\
\hline $\begin{array}{l}20,8 \\
20,8 \\
20,8 \\
20,8 \\
20,8 \\
20,8 \\
20,8 \\
21,0 \\
21,0 \\
21,0 \\
20,8\end{array}$ & $\begin{array}{l}24,9 \\
23,2 \\
22,9 \\
21,5 \\
21,5 \\
23,0 \\
20,7 \\
23,6 \\
23,0 \\
21,6 \\
20,1\end{array}$ & $\begin{array}{l}4,1 \\
2,4 \\
2,1 \\
0,7 \\
0,7 \\
2,2 \\
0,1 \\
2,6 \\
2,0 \\
0,6 \\
0,7\end{array}$ & $\begin{array}{l}x \\
x \\
x \\
x \\
x \\
x \\
\\
x \\
x \\
x\end{array}$ & $x$ & $\begin{array}{r}19,72 \\
11,53 \\
10,09 \\
3,36 \\
3,36 \\
10,57 \\
0,47 \\
12,38 \\
9,52 \\
2,86 \\
3,36\end{array}$ & $\begin{array}{l}13,92 \\
13,92 \\
13,92 \\
13,92 \\
13,92 \\
13,92 \\
13,92 \\
13,92 \\
13,92 \\
13,92 \\
13,92\end{array}$ & $\begin{array}{l}x \\
x \\
x \\
x \\
x \\
x \\
x \\
x \\
x \\
x \\
x\end{array}$ & $x$ & $\begin{array}{r}\text { 3. } 1.74 \\
11.1 .74 \\
18.1 .74 \\
24.1 .74 \\
1.2 .74 \\
\text { 8. 2. } 74 \\
14.2 .74 \\
\text { 22. 2. } 74 \\
\text { 8. 3. } 74 \\
15.3 .74 \\
15.3 .74\end{array}$ \\
\hline
\end{tabular}




\section{Richtigkeitsk on trolle}

Als Richtigkeit bezeichnet man die Abweichung des gefundenen Wertes (= Istwert) vom wahren Wert (= Sollwert). Aus dem an einer Kontrollprobe (z. B. StandardHuman-Plasma Behring) ermittelten Istwert kann als Zahlenwert für die Richtigkeit die prozentuale Abweichung vom Sollwert errechnet werden (12):

prozentuale Abweichung $=\frac{(\text { Sollwert }- \text { Istwert })}{\text { Sollwert }} \times 100$

Wie im folgendem gezeigt wird, ist die Richtigkeit als Zuverlässigkeitskriterium für Gerinnungsanalysen nur bedingt anwendbar $(10,11)$. In Tabelle 2 sind als Bejspiel die prozentualen Abweichungen für die Plasmathrombinzeit errechnet. Es werden nicht unerhebliche Abweichungen beobachtet, die an einem Tag über dem Dreifachen der Standardabweichung von Tag zu Tag (ausgedrückt als Variationskoeffizient), mehrerenorts in dessen annähernder Größenordnung liegen. Es sei erwähnt, daß durch unterschiedliche, auch, pathologische Kontrollplasmen, die Forderung einer Kontrolle des gesamten Konzentrationsbereiches erfült werden kann.

\section{Literaturverzeichnis}

1. Tonks, D. B. (1963), Clin. Chem. 9, 217-233.

2. Stamm, D. \& Büttner, H. (1969), diese Z. 7, 393-564.

3. Wooton, I. D. P. (1956), Clin. Chem. 2, 296-301.

4. Deut. Ärzteblatt 71, 911-912 u, 959-965 (1974); 68, 2228-2231 (1971).

5. Perlick, E. \& Bergmann, A. (1971), „Gerinnungslaboratorium in Klinik und Praxis“, S. 69-70, VEB Thieme, Leipzig.

6. Clauss, A. (1957), Acta Haematol. 17, 237-246.

7. Schultze, H. E. \& Schwick, G. (1953), Med. Welt 42, 1354-1357.

\section{Diskussion}

Die vorliegenden Untersuchungen zeigen in Ubereinstimmung mit anderen Autoren, daß eine laborinterne Präzisionskontrolle bei gerinnungsanaly tischen Globaltests durchführbar ist $(10,11)$. Mitgeteilt wurden - unseres Wissens erstmals - Präzisionsbefunde bei plasmatischen Gerinnungsfaktoren (Faktor I und VII/X), die durch wegs einen höheren Variationskoeffizienten aufwiesen als die Gruppentests. $\mathrm{Zu}$ berücksichtigen ist dabei, daß für die Plasmathrombinzeit, die Partielle Thromboplastinzeit und Faktor VII/X das Kontrollplasma auf die Testreagenzien abgestimmt war (beides Behringwerke), nicht dagegen für Fibrinogen (Reagenzien Boehringer Mannheim und Kontrollplasma Behringwerke). Jaroschik \& Nägle (13) wiesen am Beispiel des Quickwertes darauf hin, daß bei unterschiedlicher Provenienz von Standard und Reagenzien wesentlich schlechtere Präzisionsergebnisse erhal ten werden.

Eine Richtigkeitskontrolle ist bei Gerinnungsuntersuchungen nur bedingt ausführbar. Dies beruht einmal darauf, daß die Herstellung absoluter Referenzplasmen mit konstanter, genau bekannter Konzentration problematisch ist. Hinzu kommen die Schwierigkeiten von der Reagenzienseite, vor allem was die notwendige Stabilität bestimmter Gerinnungsaktivitäten betrifft.
8. Büttner, H. \& Stamm, D. (1966), diese Z. 4, 303-306.

9. Szasz, G. (1970), diese Z. 8, 212-217.

10. Drescher, K. H. (1969), Ärztl. Lab. 15, 245-358.

11. Parr, D. (1969), Ärtl. Lab. 15, 1-7.

12. Behringwerke A. G., Durchführung der Qualitätskontrolle klinisch-chemischer Analy sen.

13. Jaroschik, K. \& Nägle, S. (1973) diese Z. 11, 107-113.

14. Wisser, H. \& Knoll, E. (1974), Med. Klin. 69, 1287-1296.
Dr. med. Heinrich Zöller

87 Würzburg

Medizinische Poliklinik der Universität Klinikstraße 8 


\section{.}

